\title{
On certain integral Schreier graphs of the symmetric group
}

\author{
Paul E. Gunnells * \\ Department of Mathematics and Statistics \\ University of Massachusetts \\ Amherst, Massachusetts, USA \\ gunnells@math.umass . edu \\ Richard A. Scott ${ }^{\dagger}$ \\ Department of Mathematics and Computer Science \\ Santa Clara University \\ Santa Clara, California, USA \\ rscott@math.scu.edu \\ Byron L. Walden \\ Department of Mathematics and Computer Science \\ Santa Clara University \\ Santa Clara, California, USA \\ bwalden@math.scu.edu
}

Submitted: Feb 17, 2006; Accepted: May 3, 2007; Published: May 31, 2007

Mathematics Subject Classification: 05C25, 05C50

\begin{abstract}
We compute the spectrum of the Schreier graph of the symmetric group $S_{n}$ corresponding to the Young subgroup $S_{2} \times S_{n-2}$ and the generating set consisting of initial reversals. In particular, we show that this spectrum is integral and for $n \geq 8$ consists precisely of the integers $\{0,1, \ldots, n\}$. A consequence is that the first positive eigenvalue of the Laplacian is always 1 for this family of graphs.
\end{abstract}

*Supported in part by NSF grant DMS 0401525.

†Supported in part by a Presidential Research Grant from Santa Clara University. 


\section{Introduction}

Given a group $G$, a subgroup $H \subset G$, and a generating set $T \subset G$, we let $X(G / H, T)$ denote the associated Schreier graph: the vertices of $X(G / H, T)$ are the cosets $G / H$ and two cosets $a H$ and $b H$ are connected by an edge whenever $a H=t b H$ and $t \in T$. We shall assume that $T$ satisfies $t \in T \Leftrightarrow t^{-1} \in T$ so that $X(G / H, T)$ can be regarded as an undirected graph (with loops). The main result of this article is the following.

Theorem 1.1. Let $S_{n}$ be the symmetric group on $n$ letters, let $H_{n}$ be the Young subgroup $S_{2} \times S_{n-2} \subset S_{n}$, and let $T_{n}=\left\{w_{1}, \ldots, w_{n}\right\}$ where $w_{k}$ denotes the involution that reverses the initial interval $1,2, \ldots, k$ and fixes $k+1, \ldots, n$. Then for $n \geq 8$, the spectrum of the Schreier graph $X\left(S_{n} / H_{n}, T_{n}\right)$ consists precisely of the integers $0,1, \ldots, n$.

The full spectrum, complete with multiplicities, is given in Theorem 7.2 and seems interesting in its own right. There are, however, some connections with results in the literature that are worth mentioning. Given a graph $X$, let $\lambda=\lambda(X)$ denote the difference between the largest and second largest eigenvalue of the adjacency matrix. For a connected graph, $\lambda$ coincides with the first positive eigenvalue of the Laplacian and is closely related to certain expansion coefficients for $X$. In particular, one way to verify that a given family of graphs has good expansion properties is to show that $\lambda$ is uniformly bounded away from zero (see, e.g., [Lu2]).

Given a group $G$ and generating set $T \subset G$, we denote by $X(G, T)$ the corresponding Cayley graph. Several papers in the literature address spectral properties of $X\left(S_{n}, T_{n}\right)$ for certain classes of subsets $T_{n}$. In the case where $T_{n}$ is the set of transpositions $\{(1,2),(2,3), \ldots,(n-1, n)\}$, i.e., the Coxeter generators for $S_{n}$, the entire spectrum is computed by Bacher [Ba]. Here $\lambda=2-2 \cos (\pi / n)$, which approaches zero as $n$ gets large. On the other hand, in the case where $T_{n}$ is the more symmetric generating set $\{(1,2),(1,3), \ldots,(1, n)\}$, the eigenvalue gap $\lambda$ is always $1([\mathrm{FOW}, \mathrm{FH}])$. In $[\mathrm{Fr}]$, it is shown that among Cayley graphs of $S_{n}$ generated by transpositions, this family is optimal in the sense that $\lambda \leq 1$ for any set $T_{n}$ consisting of $n-1$ transpositions.

In applications, one typically wants an expanding family with bounded degree, meaning there exists some $k$ and some $\epsilon>0$ such that every graph in the family has $\lambda \geq \epsilon$ and vertex degrees $\leq k$. In [Lu1] Lubotzky poses the question as to whether Cayley graphs of the symmetric group can contain such a family. When restricting $T_{n}$ to transpositions, this is impossible, since one needs at least $n-1$ transpositions to generate $S_{n}$. In [Na] the case where $T_{n}$ is a set of "reversals" (permutations that reverse the order of an entire subinterval of $\left.\{1,2, \ldots, n\}^{1}\right)$ is considered. Although any $S_{n}$ can be generated by just three reversals, it is shown in $[\mathrm{Na}]$ that if $T_{n}$ is a set of reversals with $\left|T_{n}\right|=o(n)$, then $\lambda \rightarrow 0$ as $n \rightarrow \infty$. Hence, among Cayley graphs of $S_{n}$ generated by reversals, one obtains a negative answer to Lubotzsky's question.

The argument in $[\mathrm{Na}]$ proves the stronger result that certain Schreier graphs of the symmetric group generated by reversals cannot form an expanding family. It is well-known

\footnotetext{
${ }^{1}$ In the context of Coxeter groups, reversals are the elements of longest length in the irreducible parabolic subgroups of $S_{n}$.
} 
(and easy to see) that the spectrum of any Schreier graph $X(G / H, T)$ is a subset of the spectrum of the Cayley graph $X(G / T)$, hence if a collection of Cayley graphs forms an expanding family, so does any corresponding collection of Schreier graphs. In particular, [Na] considers the Schreier graphs corresponding to $H_{n}=S_{2} \times S_{n-2} \subset S_{n}$, and shows that if $T_{n}$ is a set of reversals and $\left|T_{n}\right|=o(n)$ then even for the Schreier graphs $X\left(S_{n} / H_{n}, T_{n}\right)$, one has $\lambda \rightarrow 0$ as $n \rightarrow \infty$.

The elements $w_{1}, \ldots, w_{n}$ in the theorem above are, in fact, reversals; $w_{k}$ flips the initial interval $1,2, \ldots, k$ and fixes $k+1, \ldots, n$. Hence, in addition to providing another example of a Schreier graph whose spectrum can be computed (with a nice explicit form), our theorem shows that the Schreier graphs $X\left(S_{n} / H_{n}, T_{n}\right)$ satisfy $\lambda=1$ for all $n$. In particular, the bound $\left|T_{n}\right|=o(n)$ in $[\mathrm{Na}]$ is essentially sharp. Empirical evidence suggests that the corresponding Cayley graphs $X\left(S_{n}, T_{n}\right)$ with $T_{n}=\left\{w_{1}, \ldots, w_{n}\right\}$ also have $\lambda=1$ for all $n$, but our methods do not extend to prove this.

\section{Preliminaries}

Let $S_{n}$ be the group of permutation of the set $\{1,2, \ldots, n\}$ and let $T_{n} \subset S_{n}$ be the set of reversals $\left\{w_{1}, \ldots, w_{n}\right\}$ given by

$$
w_{k}(i)= \begin{cases}k+1-i & \text { if } 1 \leq i \leq k \\ i & \text { if } k<i \leq n\end{cases}
$$

Let $V_{n}$ be the set consisting of 2 -element subsets $\{i, j\} \subset\{1,2, \ldots, n\}(i \neq j)$. We define the graph $X_{n}$ to be the graph with vertex set $V_{n}$ and an edge joining $\{i, j\}$ to $\left\{w_{k}(i), w_{k}(j)\right\}$ for each $k \in\{1, \ldots, n\}$. Since each $w_{k}$ is an involution, $X_{n}$ can be regarded as an undirected graph. Moreover, $X_{n}$ has loops: the vertex $\{i, j\}$ has a loop for every $w_{k}$ that fixes or interchanges $i$ and $j$. We adopt the standard convention that a loop contributes one to the degree of a vertex. Thus, $X_{n}$ is an $n$-regular graph with $\left(\begin{array}{l}n \\ 2\end{array}\right)$ vertices. The first few graphs (with loops deleted) are shown in Figure 1.

Remark 2.1. The group $S_{n}$ acts transitively on the set $V_{n}$ and the stabilizer of $\{1,2\}$ is the subgroup $S_{2} \times S_{n-2}$. It follows that $V_{n}$ can be identified with the quotient $S_{n} / S_{2} \times S_{n-2}$, and that the graph $X_{n}$ coincides with the Schreier graph $X\left(S_{n} / S_{2} \times S_{n-2}, T_{n}\right)$ described in the introduction.

Let $W_{n}=L^{2}\left(V_{n}\right)$ be the real inner product space of functions on $V_{n}$. Given $x \in W_{n}$ we shall often write $x_{i j}$ instead of $x(\{i, j\})$, and use the following format to display $x$ :

$$
x=\begin{array}{ccccc}
x_{12} & & & & \\
x_{23} & x_{13} & & & \\
x_{34} & x_{24} & x_{14} & & \\
\vdots & & & \ddots & \\
x_{n-1, n} & & \ldots & & x_{1, n}
\end{array}
$$



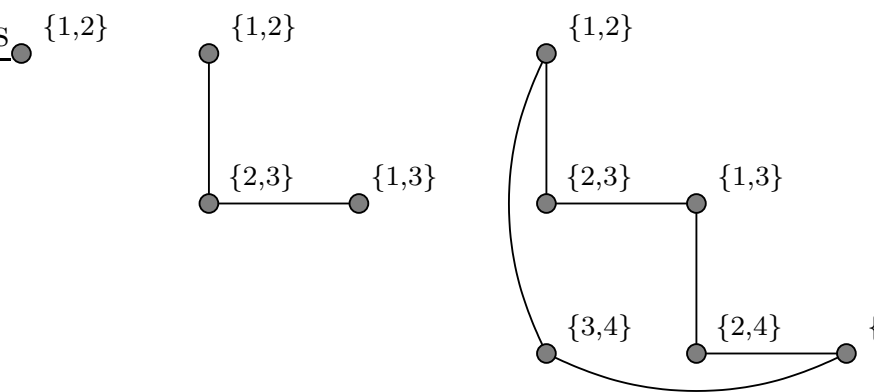

$\{1,4\}$

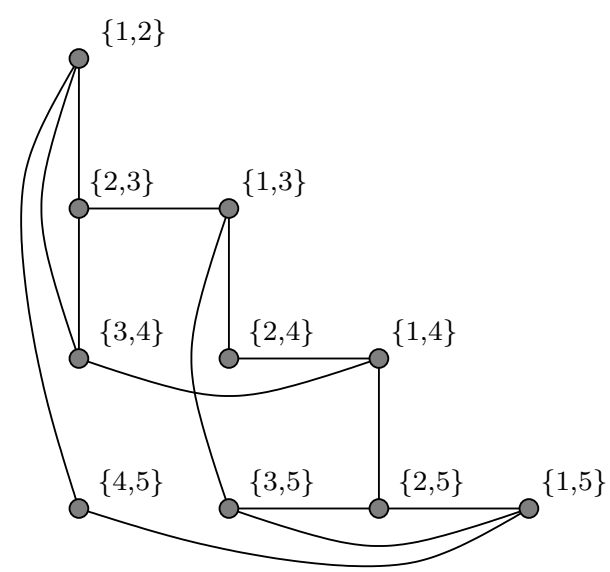

Figure 1:

Let $A=A\left(X_{n}\right): W_{n} \rightarrow W_{n}$ be the adjacency operator

$$
(A x)(\{i, j\})=\sum_{k=1}^{n} x\left(\left\{w_{k}(i), w_{k}(j)\right\}\right) .
$$

$A$ is symmetric, hence diagonalizable.

\section{The involution}

A glance at the examples in Figure 1 suggests that the graphs $X_{n}$ are symmetric about a diagonal line (from the bottom left to the top right). To prove this is indeed the case, we let $\iota$ be the involution on the vertex set $V=V_{n}$ obtained by reflecting across this diagonal line, i.e.,

$$
\iota:\{i, j\} \longmapsto\{i, n+i-j+1\}
$$

for $i<j$.

It will be convenient to picture a vertex $\{i, j\} \in V$ as a row of $n$ boxes with balls in the boxes $i$ and $j$. Assuming $i<j$, we call them the left and right balls, respectively. There are $i-1$ boxes to the left of the left ball, $j-i-1$ boxes between the two balls, and $n-j$ boxes to the right of the right ball.

We shall say that a reversal $w_{k}$ moves a ball in the $i$ th box if $i$ is contained in the interval $[1, k]$. We say that $w_{k}$ fixes a ball in the $i$ th box if $i>k$. (It may be helpful to think of $w_{k}$ as lifting up the boxes in positions from 1 to $k$, reversing them, and then putting them back down. Thus, $w_{k}$ "moves" balls in boxes 1 through $k$, even though when $k$ is odd, the ball in position $(k+1) / 2$ does not change its position.) A vertex $\{i, j\}$ determines a partition of the set $T=\left\{w_{1}, w_{2}, \ldots, w_{n}\right\}$ into three types (Figure 2):

1. Those $w_{k}$ fixing both balls (type 1 ). There are $i-1$ of these. 
2. Those $w_{k}$ moving the left ball and not the right (type 2). There are $j-i$ of these.

3. Those $w_{k}$ moving both balls (type 3 ). There are $n-j+1$ of these.

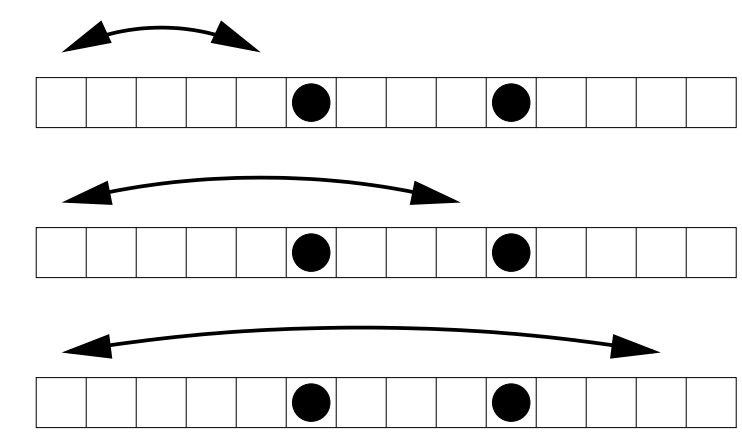

Figure 2: The three types of reversals

This also gives a partition of the set of neighbors of $\{i, j\}$ into types. Namely, a neighbor $u$ of $v$ has type 1,2 , or 3 (respectively) if $u=w \cdot v$ and $w$ has type 1 , 2, or 3 (resp.). Given $v \in V$, write $N_{p}(v)$ for the multiset of neighbors of $v$ of type $p$. (Thus for example $N_{1}(\{i, j\})$ consists of $i-1$ copies of $\{i, j\}$. We need multisets to keep track of multiple edges. Actually this only arises for neighbors of type 1; the other two neighbor multisets are really sets.)

Proposition 3.1. Let $\iota: V \rightarrow V$ be the map

$$
\iota:\{i, j\} \longmapsto\{i, n+i-j+1\} .
$$

Then $\iota$ maps $N_{1}(v)$ bijectively onto $N_{1}(\iota(v))$, and $N_{2}(v)$ bijectively onto $N_{3}(\iota(v))$. Thus $\iota$ is an involution of $X_{n}$.

Proof. We can describe the action of $\iota$ as follows: If there are $a$ boxes between the left and right balls of $v$, then the left balls of $v$ and $\iota(v)$ are in the same positions, and there are $a$ boxes to the right of the right ball of $\iota(v)$. Hence $N_{1}(v)$ is in bijection with $N_{1}(\iota(v))$, since $\iota$ keeps the left ball fixed and moves the right ball to a new "right" position.

Consider the type 2 neighbors of $v=\{i, j\}$. There are $j-i$ such neighbors. In all of them the right ball is in the same position as that of $v$ (so there are $n-j$ boxes to the right of the right ball). However the left balls of these neighbors occupy successively boxes $1, \ldots, j-i$.

Applying $\iota$ to these vertices produces a set $S$ of vertices with left ball in boxes $1, \ldots, j-$ $i$, and with $n-j$ boxes between the left and right balls. We claim that $S$ is exactly $N_{3}(\iota(v))$. First observe that $N_{3}(\iota(v))$ has the same cardinality as $S$. Also, the vertices in $N_{3}(\iota(v))$ have their left balls in positions $1, \ldots, j-i$. Finally, there are always $n-j$ boxes in between the left and right balls of elements of $N_{3}(\iota(v))$, since that is the number of boxes between the left and right balls of $\iota(v)$. This completes the proof. 
By a slight abuse of notation, we also let $\iota$ denote the induced involution on $W_{n}=$ $L^{2}(V)$. Since $\iota$ is an automorphism of $X_{n}$, it commutes with the adjacency operator $A_{n}$, hence it restricts to an involution on each eigenspace. It follows that each eigenspace can be further decomposed into odd and even subspaces. To simplify formulas later on, it will be convenient to double the standard even and odd orthogonal projections; hence we define the odd part of $x \in W_{n}$ to be $x^{-}=x-\iota(x)$ and the even part to be $x^{+}=x+\iota(x)$.

Remark 3.2. In terms of the representation for $x \in W_{n}$ in (1) the involution $\iota$ flips the diagram about the anti-diagonal, hence odd eigenvectors are antisymmetric with respect to this flip and even eigenvectors are symmetric.

\section{Standard eigenvectors}

Let $Y_{n}$ be the graph with vertex set $I_{n}=\{1,2, \ldots, n\}$ and an edge joining $i$ to $w_{k}(i)$ for each $w_{k} \in T_{n}$. Let $U_{n}$ be the inner product space $L^{2}\left(I_{n}\right)$ of functions on $I_{n}$, and let $B_{n}: U_{n} \rightarrow U_{n}$ be the adjacency operator for $Y_{n}$. We identify $U_{n}$ with $\mathbf{R}^{n}$ via the isomorphism

$$
u \mapsto(u(1), \ldots, u(n)) .
$$

Proposition 4.1. The following tables gives a basis of eigenvectors for $B_{n}$. That is, each $u(m, n)$ is an eigenvector in $B_{n}$ with corresponding eigenvalue $m$.

\begin{tabular}{|c|c|c|c|}
\hline $\begin{array}{c}n \text { even } \\
m\end{array}$ & $u(n, m)$ & $\begin{array}{c}n \text { odd } \\
m\end{array}$ & $u(n, m)$ \\
\hline 0 & $(1-n, 1,1, \ldots, 1,1,1)$ & 0 & $(1-n, 1,1, \ldots, 1,1,1)$ \\
\hline 1 & $(0,3-n, 1, \ldots, 1,1,0)$ & 1 & $(0,3-n, 1, \ldots, 1,1,0)$ \\
\hline$n / 2-1$ & $(0, \ldots, 0,-1,1,0,0, \ldots, 0)$ & $(n-3) / 2$ & $(0, \ldots, 0,-2,1,1,0, \ldots, 0)$ \\
\hline$n / 2+1$ & $(0, \ldots, 0,1,1,-2,0, \ldots, 0)$ & $(n+1) / 2$ & $(0, \ldots, 0,0,1,-1,0, \ldots, 0)$ \\
\hline$n-2$ & $(0,0,1, \ldots, 1,4-n, 0)$ & $n-2$ & $(0,0,1, \ldots, 1,4-n, 0)$ \\
\hline$n-1$ & $(0,1,1, \ldots, 1,1,2-n)$ & $n-1$ & $(0,1,1, \ldots, 1,1,2-n)$ \\
\hline$n$ & $(1,1,1, \ldots, 1,1,1)$ & $n$ & $(1,1,1, \ldots, 1,1,1)$ \\
\hline
\end{tabular}

Proof. The first two cases $n=2,3$ can be checked directly. In general, the induced graph $Y_{n}^{\prime}=Y_{n}-\{1, n\}$ is isomorphic to $Y_{n-2}$ with an extra loop added to each vertex (with the isomorphism given on vertices by $i \mapsto i-1)$. The vertex 1 in $Y_{n}$ has one loop and is connected to each vertex of $Y_{n}^{\prime}$ by a single edge; the vertex $n$ has $n-1$ loops and is connected only to vertex 1 . It follows that any eigenvector for $Y_{n}^{\prime}$ except for the constant one $(1,1, \ldots, 1)$ can be extended to an eigenvector for $Y_{n}$ by setting $e_{1}=e_{n}=0$. Since $Y_{n}^{\prime}$ has one more loop on each vertex than $Y_{n-2}$, the corresponding eigenvalue increases by one. This produces all of the eigenvectors in the table except those for $\lambda=0, n-1, n$, and 
these can be checked directly (note that they are all extensions of the constant eigenvector in $\left.Y_{n-2}\right)$.

It will be convenient to break up these eigenvectors into three types:

- the trivial type $u(n, n)=(1, \ldots, 1)$,

- the left type (for $0 \leq m<\frac{n}{2}-1$ )

$$
u(n, m)=(\underbrace{0, \cdots, 0}_{m}, 1+2 m-n, \underbrace{1, \cdots, 1}_{n-1-2 m}, \underbrace{0, \cdots, 0}_{m})
$$

- the right type (for $\frac{n+1}{2} \leq m \leq n-1$ )

$$
u(n, m)=(\underbrace{0, \cdots, 0}_{n-m}, \underbrace{1, \cdots, 1}_{2 m-n}, n-2 m, \underbrace{0, \cdots, 0}_{n-1-m})
$$

Letting $\phi: U_{n} \rightarrow W_{n}$ be the natural map $\phi(u)(i, j)=u(i)+u(j)$, it is easy to see that $\phi \circ A_{n}=B_{n} \circ \phi$. It follows that each of the eigenvectors $u(n, m)$ for $B_{n}$ gives rise to a corresponding $m$-eigenvector $w(n, m)$ for $A_{n}$, i.e.,

$$
w(n, m)(\{i, j\})=u(n, m)(i)+u(n, m)(j) .
$$

In the case of the trivial type $u(n, n)$, the corresponding eigenvector $w(n, n)$ is the constant vector and has eigenvalue $n$. For the other types, it is easier to describe the odd and even parts $w(n, m)^{ \pm}$. When $u(n, m)$ is of the left type the eigenvectors $w(n, m)^{ \pm}$are shown in Figures 6, 8, and 9 (note that when $m=0$, the odd part $w(n, m)^{-}$is zero). When $u(n, m)$ is of the right type the eigenvectors $w(n, m)^{ \pm}$are shown in Figures 7,10 , and 11.

\section{Zero eigenvectors}

In this section, we give explicit formulas for $n-3$ linearly independent 0 -eigenvectors in $W_{n}$. One of these is the standard (even) eigenvector $w(n, 0)^{+}$described above. We separate the remaining ones into even and odd types. The odd ones will be denoted by $z(n, p)^{-}$(where $p$ is any integer such that $2 \leq p \leq \frac{n-1}{2}$ ) and are shown in Figure 12 . The even ones, denoted by $z(n, p)^{+}$(where $1 \leq p \leq \frac{n-4}{2}$ ) are shown in Figure 13 (in the case $1 \leq p \leq \frac{n-4}{3}$ ) and Figure 14 (in the case $\frac{n-4}{3} \leq p \leq \frac{n-4}{2}$ ). Note, all dotted lines indicate an arithmetic progression (possibly constant) between the endpoints. A number under a dotted line indicates the number of terms in the sequence - including both endpoints. The proofs that these are, indeed, null-vectors is a computation that breaks up into a finite number of cases (based on the form of the array). We give an example of how this computation works, and leave the remaining verifications to the interested reader. 


\section{Theorem 5.1.}

1. The sum of the entries along any column or diagonal of the vectors $z(n, p)^{ \pm}$vanishes.

2. The vectors $z(n, p)^{ \pm}$lie in the kernel of the adjacency matrix.

Proof. Statement (1) is a trivial computation. Statement (2) is verified by explicitly computing the action of the adjacency matrix $A$ on $z(n, p)^{ \pm}$. We give the proof for the antisymmetric vectors $z(n, p)^{-}$; the proof for the symmetric vectors is only slightly more complicated and involves no new ideas.

The first step is to break $z=z(n, p)^{-}$up into regions suggested by the structure shown in Figure 12:

(A) the upper triangle $\left\{z_{i j} \mid j \leq p-1\right\}$;

(B) the row of $(p-n)$ 's $\left\{z_{i j} \mid j=p, i>1\right\}$;

(C) the $x$ at $i=1, j=p$;

(D) the rectangle of 1 's $\left\{z_{i j} \mid p+1 \leq j \leq n+1-p, ; 1 \leq j-i \leq p-2\right\}$;

(E) the column of $(2-p)$ 's $\left\{z_{i j} \mid p+1 \leq j \leq n+1-p, j-i=p-1\right\}$;

(F) the row of $(p-1)$ 's $\left\{z_{i j} \mid j=n+2-p, 1 \leq j-i \leq p-2\right\}$;

(G) the lower box of 0's $\left\{z_{i j} \mid n+3-p \leq j \leq n, 1 \leq j-i \leq p-2\right\}$;

(H) the central triangle of 0's $\left\{z_{i j} \mid p+1 \leq j \leq n+1-p, p \leq i-j\right\}$;

(I) the 0 appearing below $2-p$ and to the right of $p-1$.

By symmetry it suffices to show that any component of $A z$ in any of these regions vanishes. We do this by breaking the sum $(A z)_{i j}$ into three parts, corresponding to the three types of reversals as in the proof of Proposition 3.0.3. We will be somewhat brief and leave most details to the reader.

Case A: Type 1 and 2 reversals contribute 0 to $(A z)_{i j}$. Type 3 reversals also contribute 0 since the sum of all entries in a column is 0 .

Case B: There are $i-1$ type 1 reversals, each contributing $p-n$. The type 2 reversals contribute $x$ and then $j-i-1$ copies of $p-n$. The total from these two types is 0 , and it is not hard to see that the type 3 reversals contribute $(p-n)+(n-2 p+1)+p-1=0$.

Case C: There are no reversals of type 1 . Reversals of type 2 contribute $x+(p-$ $2)(p-n)$. Reversals of type 3 give $x+(p-2)(1-p)+(2-p)(n-2 p+1)$, and the total is 0 .

Case D: This region, together with E, G, and H, are the most complicated. The region $\mathrm{D}$ must be subdivided into two subregions, an isosceles right triangle and a trapezoid (Figure 3). The top edge of the trapezoid is $i=p$. In the trapezoid, type 1 gives $i-1$, type 2 gives 0 , and type 3 gives $(p-n)+n-p-i+1$, for a total of zero. 
In the triangle, type 2 reversals give a nonzero contribution; the contribution of type 1 and type 2 depends on how far $z_{i j}$ is above the diagonal. If $z_{i j}$ is on the $d$ th diagonal band, then $i=p-d$, and the contributions from type 1 and type 2 are respectively $i-1=p-d-1$ and $1-p+d$, which total 0 . The type 3 reversals give a whole column sum, and thus the total is 0 .

Case E: As in case D we must consider two cases, an upper rectangle and a lower rectangle; moreover the upper rectangle must be broken into boxes exactly as $\mathrm{D}$ is broken into bands (Figure 3). The computations are essentially the same as for case D.
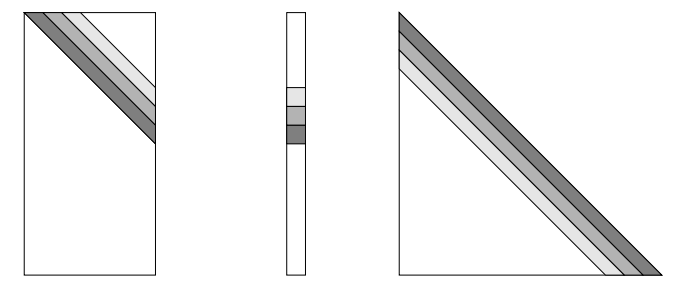

Figure 3: The trapezoid and triangle for case D, with three diagonal bands. Three boxes for case E. Three bands for case $\mathrm{H}$.

Case F: Type 1 gives $(i-1)(p-1)$, type 2 gives $-x+(j-i-1)(p-2)$, and type 3 gives $(p-n)+(j-i-1)$, which sum to 0 .

Case G: Consider Figure 4. By symmetry, the central diagonal band is forced to vanish, and we only have to check the entries above the diagonal. Temporarily number the entries in the square so that $z_{s t}$ denotes the entry on row $s$ up from the bottom and column $t$ from the left. All type 1 contributions vanish. The type 2 contributions are the sum of $t$ rightmost entries in the row containing $z_{s t}$. The type 3 contributions are the sum of the $s$ entries from the top of the column containing $z_{s t}$. It is easy to see that these cancel, and so the total is 0 .

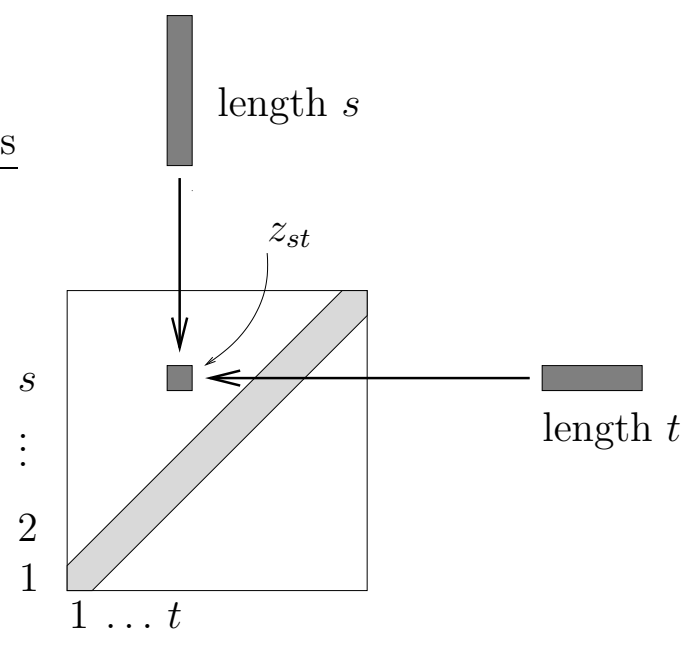

Figure 4: Case G 
Case H: This triangle must be broken into bands along the oblique edge, just as for case D (Figure 3). In this case the contribution of the type 1 reversals is 0 , and the contribution from type 2 exactly cancels that from type 3 .

Case I: This entry is forced to be zero by the antisymmetry.

This completes the proof.

\section{Promotion}

In this section, we describe the remaining eigenvectors. Note that the standard eigenvectors $w(n, m)$ and the zero eigenvectors $z(n, k)^{ \pm}$determine a complete basis for $W_{n}$ in dimensions $n=2,3,4$. For $n \geq 4$ we use a "promotion scheme" to create for each $m$-eigenvector in dimension $n$, a $(m+1)$-eigenvector in dimension $n+3$. The construction is based on the observation that $X_{n+3}$ contains an induced subgraph that is isomorphic to $X_{n}$, but with an extra loop added to each vertex.

Given a graph $G$, we let $G^{\circ}$ denote the same graph $G$, but with an extra loop added to each vertex. Since adding a loop to each vertex corresponds to adding 1 to the adjacency operator, the eigenvalues of $G^{\circ}$ are the same as the eigenvalues of $G$, but shifted up by 1 . Given a subset $K$ of vertices in a graph $G$, we let $G[K]$ denote the induced subgraph on the set $K$.

We partition $V_{n+3}$ into the following subsets:

$$
\begin{aligned}
& S=\{\{1,2\},\{1,3\}, \ldots\{1, n+3\}\} \cup\{\{n+2, n+3\}\} \\
& L=\{\{2,3\},\{3,4\}, \ldots,\{n+1, n+2\}\} \\
& B=\{\{2, n+3\},\{3, n+3\}, \ldots,\{n+1, n+3\}\} \\
& C=\{\{i, j\} \mid 2 \leq i<j \leq n+2, j-i \geq 2\} .
\end{aligned}
$$

With respect to our triangular representation of $X_{n+3}$ (as in Figure 1), $S$ corresponds to the vertices along the hypotenuse, $L$ corresponds to the vertices along the left side (minus the top and bottom vertices), $B$ corresponds to the vertices along the bottom row (minus

the left and right vertices), and $C$ corresponds to the "central" vertices in the interior of the triangle.

We then define bijections $\pi_{L}: L \rightarrow I_{n}, \pi_{B}: B \rightarrow I_{n}$, and $\pi_{C}: C \rightarrow V_{n}$ by

$$
\begin{aligned}
& \pi_{L}(\{i, j\})=i-1 \\
& \pi_{B}(\{i, j\})=i-1 \\
& \pi_{C}(\{i, j\})=\{i-1, j-2\} .
\end{aligned}
$$

Recall that the image sets $I_{n}$ and $V_{n}$ are the vertex sets for the graphs $Y_{n}$ and $X_{n}$, respectively. With respect to the induced subgraphs of $X_{n+3}$ corresponding to the sets $L$, $B$, and $C$, it turns out that the maps $\pi_{L}, \pi_{B}$, and $\pi_{C}$ induce graph isomorphisms (once loops are added to the target graphs). We state this precisely in the next proposition. Figure 5 illustrates the $n=4$ case. 
Proposition 6.1. $X_{n+3}-S$ decomposes into three components, $X_{n+3}[L], X_{n+3}[B]$, and $X_{n+3}[C]$. Moreover, the maps $\pi_{L}, \pi_{B}$, and $\pi_{C}$ induce graph isomorphisms $X_{n+3}[L] \cong Y_{n}^{\circ}$, $X_{n+3}[B] \cong Y_{n}^{\circ}$, and $X_{n+3}[C] \cong X_{n}^{\circ}$, respectively.

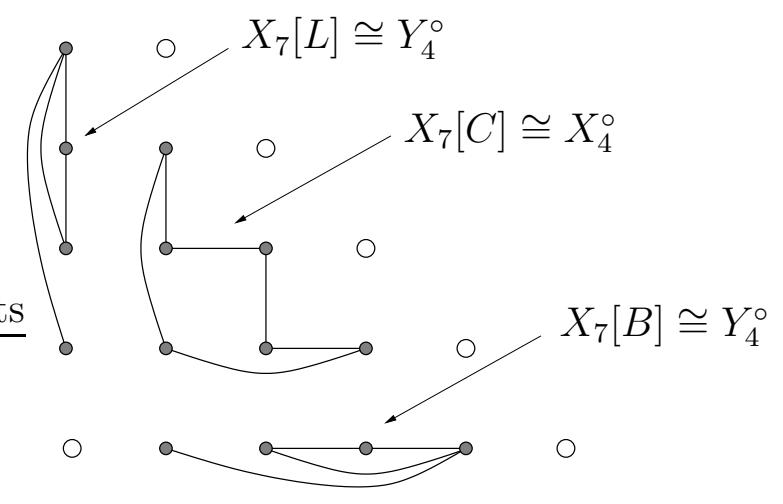

Figure 5: The induced subgraph on $V_{7}-S$ (elements of $S$ are the "open dots")

Proof. The proof is straightforward (using, for example, the "balls in boxes" interpretation as in the proof of Proposition 3.1.) We sketch the proof that $\pi_{C}$ is an isomorphism and leave the rest to the reader. Start with $v=\{i, j\} \in C$ (the vertex set of $X_{n+3}[C]$ ). We can list the neighbors of $v$ in $X_{n+3}$ that are also neighbors in $X_{n+3}[C]$ as follows.

1. All $i-1$ neighbors of type 1 .

2. All neighbors of type 2 except the one coming from the reversal $w_{i}$. Hence there are $j-i-1$ of these.

3. All neighbors of type 3 except the one coming from the reversal $w_{j}$. Hence there are $n+3-j$ of these.

Therefore the degree of $X_{n+3}[C]$ is $n+1$, which is the degree of $X_{n}^{\circ}$. Moreover, a closer look at the cases above shows that the map $\pi_{C}$ induces a bijection from $N_{p}(v)$ to $N_{p}\left(\pi_{C}(v)\right)$ where the former is understood to be the neighbors of $v=\{i, j\}$ of type $p$ computed in $X_{n+3}[C]$, and the latter is the neighbors of $\pi_{C}(v)=\{i-1, j-2\}$ of type $p$ computed in $X_{n}^{\circ}$ (we regard the extra loop in $X_{n}^{\circ}$ as type 1 ). It follows that $\pi_{C}$ is an isomorphism.

Given a vector $x \in W_{n+3}=L^{2}\left(V_{n+3}\right)$, let $\left.x\right|_{L},\left.x\right|_{B}$, and $\left.x\right|_{C}$ denote the restrictions to $L, B$, and $C$, respectively. We regard these restrictions as functions on $Y_{n}, Y_{n}$, and $X_{n}$, respectively.

Proposition 6.2. Suppose $x \in W_{n+3}$ vanishes on $S$. Then $x$ is an eigenvector of $X_{n+3}$ with eigenvalue $m$ if and only if 
(i) $\left.x\right|_{L}$ is either zero or an eigenvector of $Y_{n}$ with eigenvalue $m-1$

(ii) $\left.x\right|_{B}$ is either zero or an eigenvector of $Y_{n}$ with eigenvalue $m-1$

(iii) $\left.x\right|_{C}$ is either zero or an eigenvector of $X_{n}$ with eigenvalue $m-1$

(iv) for each (diagonal) vertex $\{1, k\}$, the corresponding row and column sums of $x$ sum to zero: i.e.,

$$
\sum_{j=1}^{k-1} x(\{j, k\})+\sum_{j=1}^{n+4-k} x(\{j, j+k-1\})=0 .
$$

Proof. The necessity of the first three conditions follows from Proposition 6.1. The necessity of (iv) follows from the fact that the vertex $v=\{1, k\}$ is adjacent precisely to those vertices in the same row and column as $v$. Hence in order for the adjacency operator to take $x$ to a vector that still vanishes on $v$, this sum must be zero. It is also clear from Proposition 6.1, that these four conditions are sufficient to guarantee that $x$ is an eigenvector of $X_{n+3}$.

We use this proposition to build the remaining eigenvectors. First suppose $\left.x\right|_{C}=0$. It follows from (iv) and the list of eigenvectors for $Y_{n}$ that the only possibilities are when $n$ is even, $m=n / 2-1$ and

$$
\left.x\right|_{B}=\left.x\right|_{L}=u(n, m)=(\underbrace{0, \cdots, 0}_{m},-1,1, \underbrace{0, \cdots, 0}_{m})
$$

We let $y(n+3)^{+}$denote this vector (it is symmetric). The vector $y(n)^{+}$is the $k=0$ case of Figure 19; it has eigenvalue $m=(n-3) / 2$.

Next suppose $\left.x\right|_{C}$ is not zero. Then it must be an eigenvector for $X_{n}$. We consider the following cases.

Case 1: $\left.x\right|_{C}$ is one of the (nonstandard) zero eigenvectors $z(n, p)^{ \pm}$. In this case, all of the row sums are zero and all of the column sums are zero, so condition (iv) will be satisfied if and only if the restrictions $\left.x\right|_{L}$ and $\left.x\right|_{B}$ are zero. Thus, for each $z(n, p)^{ \pm}$, there is a unique eigenvector $x$ in $W_{n+3}$ obtained by placing zeros down the diagonal, left side, and bottom (we shall refer to this procedure henceforth as extension by zero). The new eigenvector will have eigenvalue 1 . Moreover, since the new eigenvector will have the same row sums and column sums, we can continue to extend by zero. In general, we define $\rho^{0} z(n, p)^{ \pm}=z(n, p)^{ \pm}$and define $\rho^{k} z(n, p)^{ \pm}$(inductively) to be the extension by zero of $\rho^{k-1} z(n, p)^{ \pm}$(respectively). The new vector $\rho^{k} z(n, p)^{ \pm}$is an eigenvector in $W_{n+3 k}$ with eigenvalue $k$.

Case 2a: $\left.x\right|_{C}$ is one of the even standard eigenvectors $w(n, m)^{+}$of left type. These can be extended as shown in Figure 17. The vector shown is the $k+1$ st extension $\rho^{k+1} w(n, m)^{+}$. It is an eigenvector in $W_{n+3 k+3}$ with eigenvalue $m+k+1$. (The pattern is established after the first extension, which can be seen by removing the $k$ outside "layers" of the triangle.) 
Case 2b: $\left.x\right|_{C}$ is one of the even standard eigenvectors $w(n, m)^{+}$of right type. These can be extended as shown in Figure 18. The vector shown is the $k+2$ nd extension $\rho^{k+2} w(n, m)^{+}$, and the displayed form for this vector is valid as long as $k \leq 2 m-n$. For $k=2 m-n$, row and column sums of $\rho^{k+1} w(n, m)^{+}$will all be zero, hence for $k>2 m-n$, we define $\rho^{k+2} w(n, m)^{+}$inductively to be the extension by zero of $\rho^{k+1} w(n, m)^{+}$. Thus $\rho^{k} w(n, m)^{+}$is defined for all $k \geq 1$ and is an eigenvector in $W_{n+3 k}$ with eigenvalue $m+k$.

Case 3a: $\left.x\right|_{C}$ is one of the odd standard eigenvectors $w(n, m)^{-}$of left type. These can be extended to an eigenvector $\rho^{1} w(n, m)^{-}$in $W_{n+3}$ as shown in Figure 15. After this initial extension, the row sums and column sums are all zero (this is easy to see by inspection), hence we can continue to extend by zero. That is, we define $\rho^{k} w(n, m)^{-}$(inductively) to be the extension by zero of $\rho^{k-1} w(n, m)^{-}$for all $k \geq 2$. The new eigenvector $\rho^{k} w(n, m)^{-}$will have eigenvalue $m+k$.

Case 3b: $\left.x\right|_{C}$ is one of the odd standard eigenvectors $w(n, m)^{-}$of right type. These can be extended to an eigenvector $\rho^{1} w(n, m)^{-}$in $W_{n+3}$ as shown in Figure 16. After this initial extension, the row sums and column sums are all zero (again, easy to see by inspection), hence we can continue to extend by zero. That is, we define $\rho^{k} w(n, m)^{-}$(inductively) to be the extension by zero of $\rho^{k-1} w(n, m)^{-}$for all $k \geq 2$. The new eigenvector $\rho^{k} w(n, m)^{-}$will have eigenvalue $m+k$.

Case 4: $\left.x\right|_{C}$ is one of the even eigenvectors $y(n)^{+}$defined above. These can be extended as shown in Figure 19. The vector shown is the $k$ extension $\rho^{k} y(n)^{+}$. It is an eigenvector in $W_{n+3 k}$ and has eigenvalue $m=(n-3) / 2+k$.

\section{The main theorem}

In this section we show that the eigenvectors we have described so far are sufficient to determine a basis for $W_{n}$. Given an eigenvalue $m$ (for $A_{n}$ ), we let $W_{n}^{m}$ denote the corresponding eigenspace.

Define the numbers $a(n, m), 0 \leq m \leq n, n \geq 2$ inductively as follows:

Initial values:

$$
\begin{aligned}
& a(2,0)=0, a(2,1)=0, a(2,2)=1 \\
& a(3,0)=1, a(3,1)=0, a(3,2)=1, a(3,3)=1 \\
& a(4,0)=1, a(4,1)=2, a(4,2)=0, a(4,3)=2, a(4,4)=1 \\
& a(n, 0)=n-3, a(n, n-1)=2, a(n, n)=1 \text { for all } n \geq 5
\end{aligned}
$$

Inductive formula:

$$
a(n, m)= \begin{cases}a(n-3, m-1) & \text { if } m=\lfloor n / 2\rfloor \text { and } n \geq 5 \\ a(n-3, m-1)+3 & \text { if } m=\lfloor n / 2\rfloor-1, n \text { is odd, and } n \geq 5 \\ a(n-3, m-1)+1 & \text { if } m=\lfloor n / 2\rfloor+1, n \text { is odd, and } n \geq 5 \\ a(n-3, m-1)+2 & \text { otherwise. }\end{cases}
$$

A small table of $a(n, m)$ is given in Table 1 . 


\begin{tabular}{c|ccccccccccc} 
& 0 & 1 & 2 & 3 & 4 & 5 & 6 & 7 & 8 & 9 & 10 \\
\hline 2 & 0 & 0 & 1 & & & & & & & & \\
3 & 1 & 0 & 1 & 1 & & & & & & & \\
4 & 1 & 2 & 0 & 2 & 1 & & & & & & \\
5 & 2 & 3 & 0 & 2 & 2 & 1 & & & & & \\
6 & 3 & 3 & 2 & 1 & 3 & 2 & 1 & & & & \\
7 & 4 & 3 & 5 & 0 & 3 & 3 & 2 & 1 & & & \\
8 & 5 & 4 & 5 & 2 & 2 & 4 & 3 & 2 & 1 & & \\
9 & 6 & 5 & 5 & 5 & 1 & 4 & 4 & 3 & 2 & 1 & \\
10 & 7 & 6 & 5 & 7 & 2 & 3 & 5 & 4 & 3 & 2 & 1
\end{tabular}

Table 1: The multiplicities $a(n, m)$. Read top to bottom for increasing $n$, left to right for increasing $m$.

Proposition 7.1. For $n \geq 2$, we have $\sum_{m=0}^{n} a(n, m)=\left(\begin{array}{l}n \\ 2\end{array}\right)=\operatorname{dim} W_{n}$.

Proof. This is obvious for $n \leq 4$. For $n \geq 5$, we have

$$
\begin{aligned}
\sum_{m=0}^{n} a(n, m) & =a(n, 0)+a(n, n-1)+a(n, n)+\sum_{m=1}^{n-2} a(n, m) \\
& =(n-3)+2+1+\left[\sum_{m=1}^{n-2} a(n-3, m-1)\right]+2(n-3) \\
& =3 n-6+\left(\begin{array}{c}
n-3 \\
2
\end{array}\right) \quad \text { (by induction) } \\
& =\left(\begin{array}{l}
n \\
2
\end{array}\right) .
\end{aligned}
$$

Our main result is the following.

Theorem 7.2. The spectrum of $A_{n}$ is contained in $\{0,1, \ldots, n\}$ with eigenvalue multiplicities given by $\operatorname{dim} W_{n}^{m}=a(n, m)$. In particular, for $n \geq 8$, the set of eigenvalues is precisely $\{0,1, \ldots, n\}$.

Proof. In light of Proposition 7.1, it suffices to show that $\operatorname{dim} W_{n}^{m}=a(n, m)$. For $2 \leq$ $n \leq 4$, all of the eigenvectors are standard eigenvectors $w(n, m)$, so this can be verified directly. For $m=0$ (and all $n$ ), the vectors $z(n, p)^{ \pm}$are linearly independent and there are $a(n, 0)=n-3$ of them. For $m=n-1$ (and all $n$ ) the standard eigenvectors $w(n, n-1)^{ \pm}$ are linearly independent and there are $a(n, n-1)=2$ of them. For $m=n$ (and all $n$ ), the constant function 1 is an eigenvector with eigenvalue $n$, so $\operatorname{dim} W_{n} \geq 1=a(n, n)$.

For the remaining eigenspaces with $n \geq 5$, we proceed by induction to show that there are $a(n, m)$ linearly independent eigenvectors of the form $\rho^{k} z^{ \pm}, \rho^{k} w^{ \pm}$, or $\rho^{k} y^{+}$(where we adopt the convention $v=\rho^{0} v$ ). Any eigenvector of the form $\rho^{k} z^{ \pm}, \rho^{k} w^{ \pm}$, or $\rho^{k} y^{+}$in dimension $n-3$ can be promoted to an eigenvector (also of this form) by the promotion scheme detailed in Section 6. Since promotion preserves linear independence and shifts eigenvalues by 1 , we have by induction $\operatorname{dim} W_{n}^{m} \geq a(n-3, m-1)$. 
When $m \neq\lfloor n / 2\rfloor$ there are two new standard eigenvectors $w(n, m)^{ \pm}\left(=\rho^{0} w(n, m)^{ \pm}\right)$ to consider. These will almost always be linearly independent from the promoted eigenvectors since standard eigenvectors are not zero down the main diagonal while promoted vectors are. The only exception to this is when $n$ is odd and $m=\lfloor n / 2\rfloor+1$, in which case only the odd projection has nonzero entries on the diagonal. This gives $\operatorname{dim} W_{n}^{m} \geq a(n-3, m-1)+1=a(n, m)$ when $n$ is odd and $m=\lfloor n / 2\rfloor+1$. On the other hand, the additional vector $y(n)^{+}$adds a new eigenvector when $n$ is odd and $m=\lfloor n / 2\rfloor-1$, giving $\operatorname{dim} W_{n}^{m} \geq a(n-3, m-1)+3=a(n, m)$ in this case. We then have $W_{n}^{m} \geq a(n-3, m-1)+2=a(n, m)$ in the remaining cases, and these inequalities now all become sharp in light of the dimension count in Proposition 7.1.

\section{References}

[Ba] R. Bacher. Valeur propre minimale du laplacien de Coxeter pour le group symétrique. Journal of Algebra 167 (1994), 460-472.

[FH] J. Friedman and P. Hanlon. On the Betti numbers of chessboard complexes. J. Algebraic Combin. 8 (1998), 193-203.

[FOW] L. Flatto, A.M. Odlyzko, and D.B. Wales. Random shuffles and group representations. Annals of Probability 13 (1985), 154-178.

[Fr] J. Friedman. On Cayley graphs of the symmetric group generated by transpositions. Combinatorica 20 (2000), 505-519.

[Lu1] A. Lubotzky. Cayley graphs: eigenvalues, expanders and random walks. Surveys in combinatorics, 1995 (Stirling), 155-189, London Math. Soc. Lecture Note Ser. 218, Cambridge Univ. Press.

[Lu2] A. Lubotzky. Discrete groups, expanding graphs and invariant measures. Progress in Mathematics, vol. 125, Birkhäuser Verlag, Basel 1994.

[Na] D. Nash. Cayley graphs of symmetric groups generated by reversals. Pi Mu Epsilon Journal 12 (2005), 143-147. 


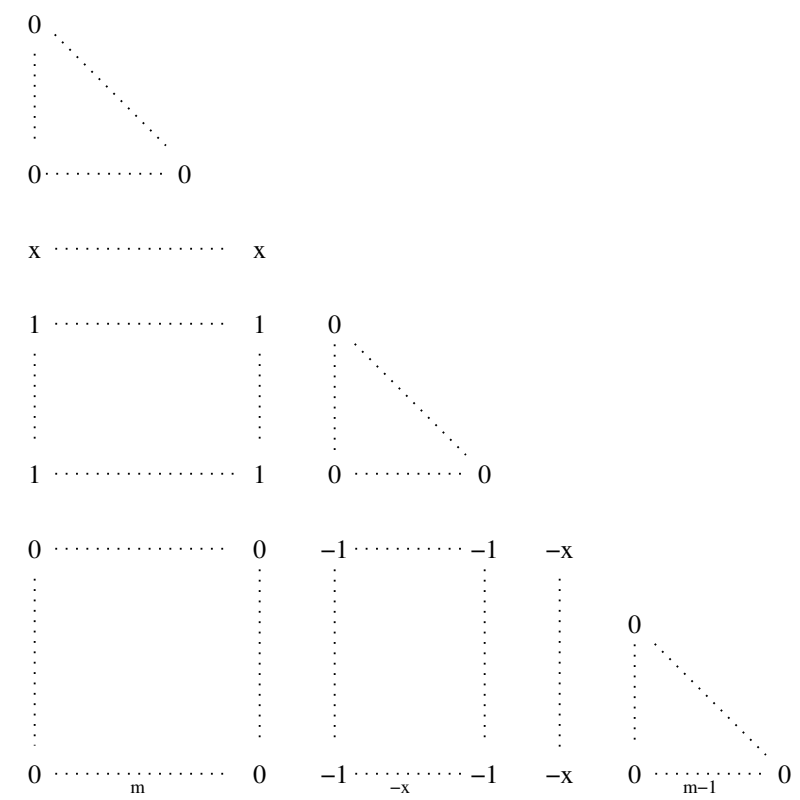

Figure 6: Left type/odd: $w(n, m)^{-}$with $x=1+2 m-n$ and $0<m<\frac{n}{2}-1$.

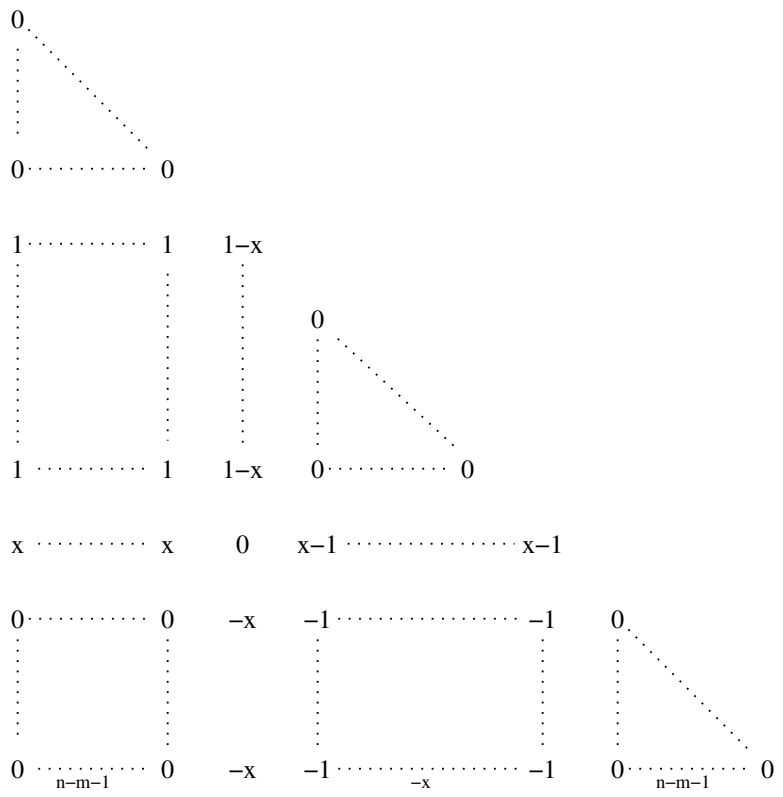

Figure 7: Right type/odd: $w(n, m)^{-}$with $x=n-2 m$ and $\frac{n+1}{2} \leq m \leq n-1$. 


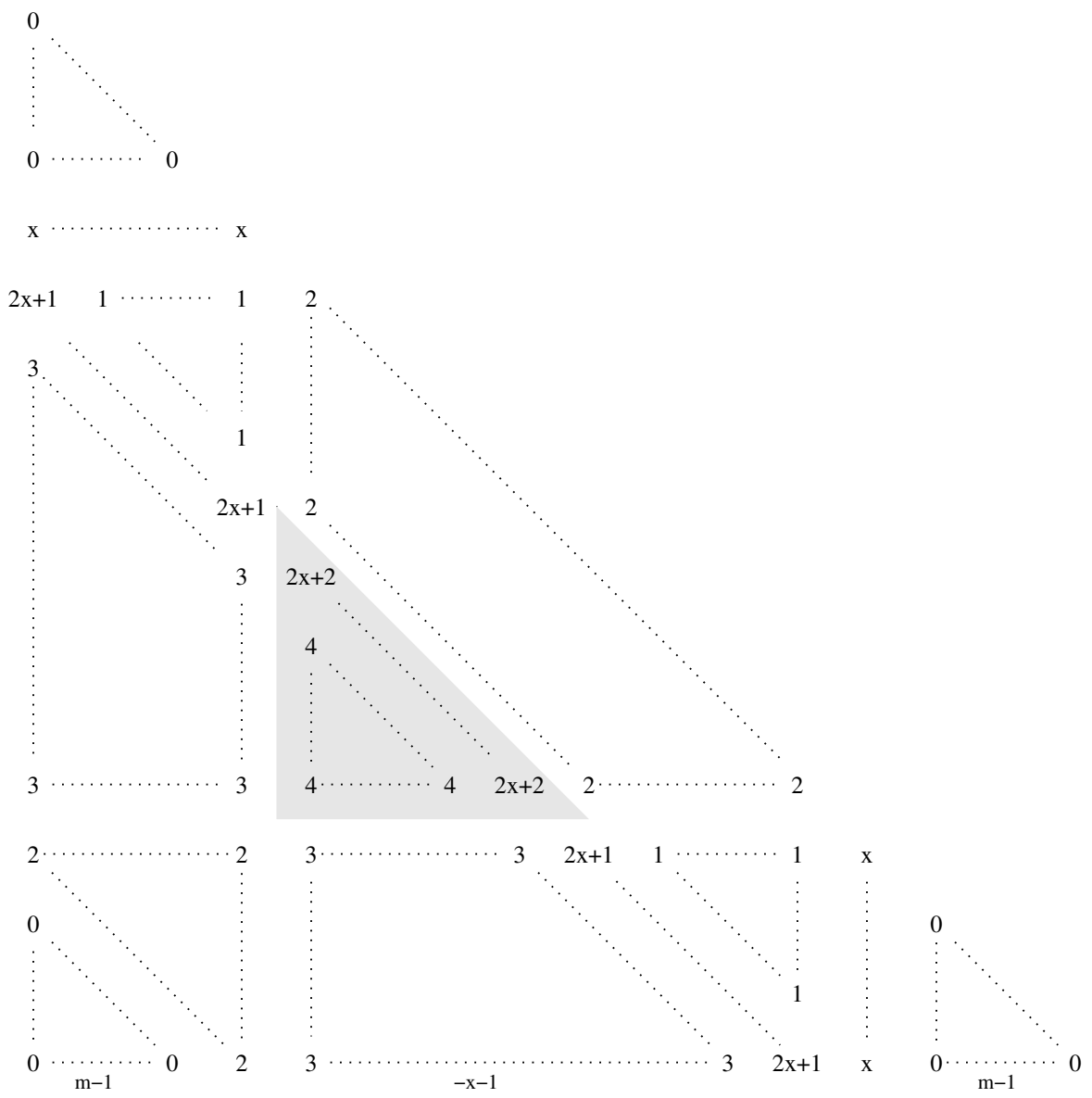

Figure 8: Left type/even, small $m: w(n, m)^{+}$with $x=1+2 m-n$ and $0 \leq m<\frac{n-1}{3}$. (The case $m=0$ reduces to the shaded triangle in the middle.) 

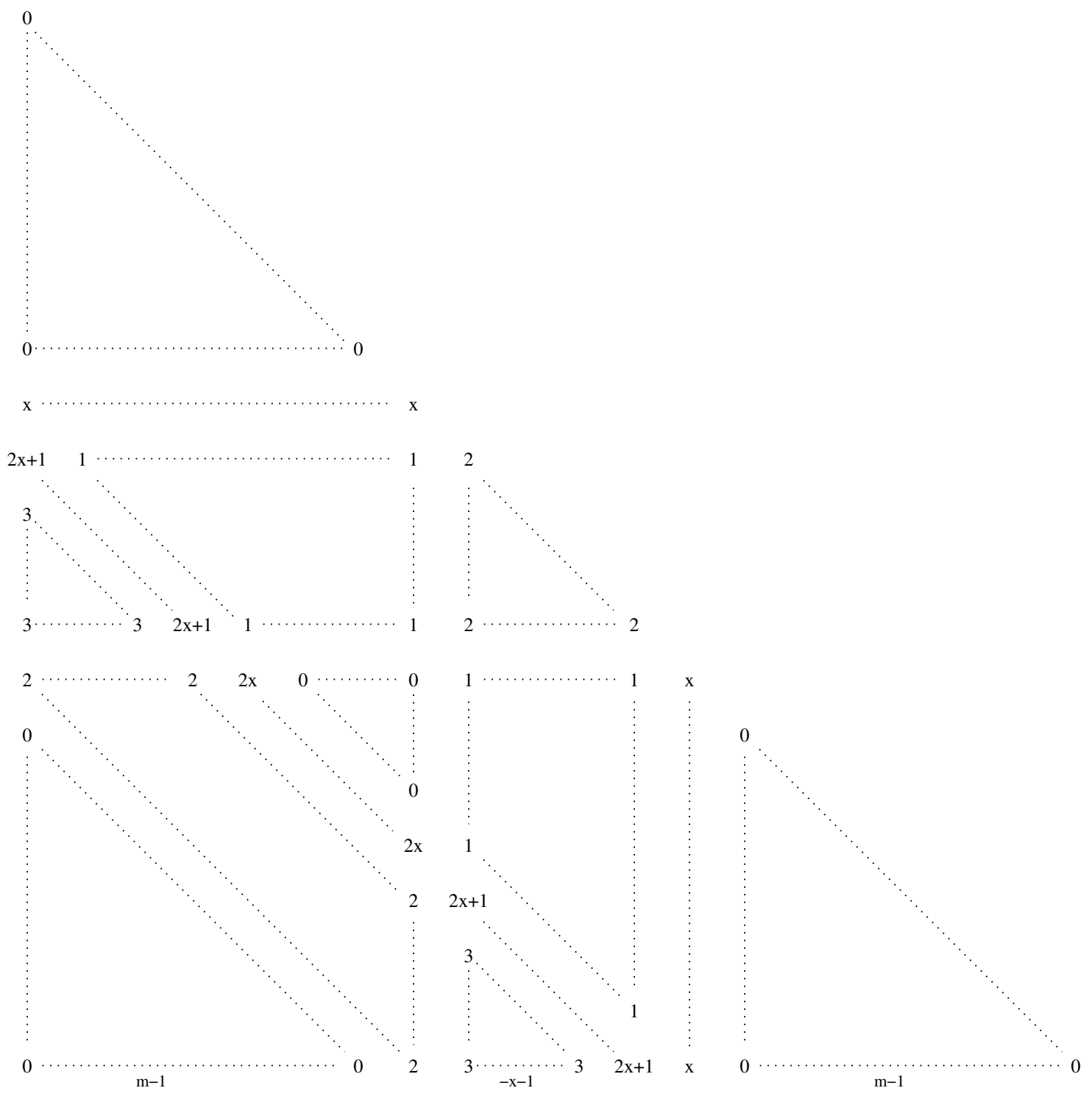

Figure 9: Left type/even, large $m: w(n, m)^{+}$with $x=1+2 m-n$ and $\frac{n-1}{3} \leq m<\frac{n}{2}-1$. 


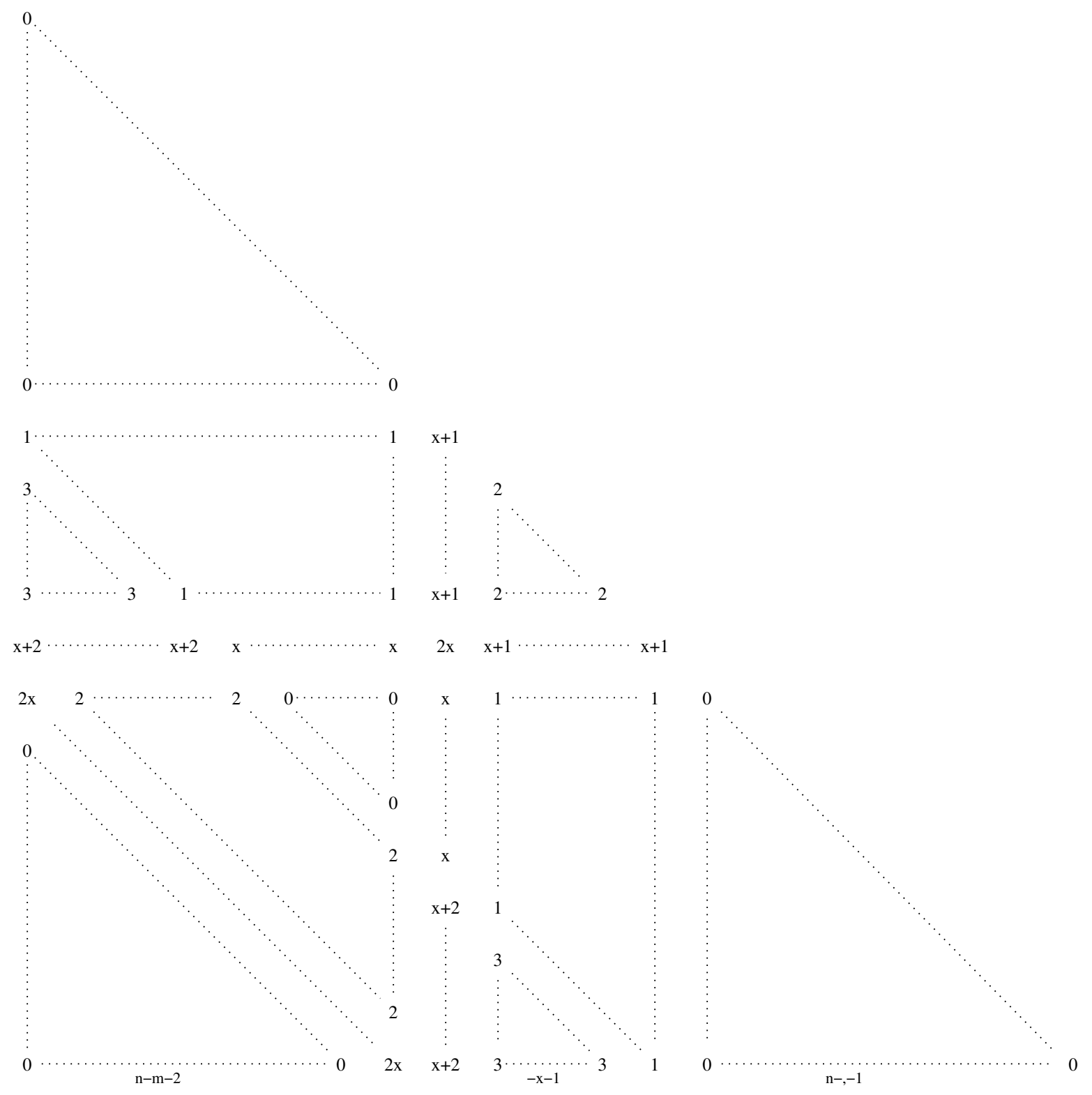

Figure 10: Right type/even, small m: $w(n, m)^{+}$with $x=n-2 m$ and $\frac{n+1}{2} \leq m \leq \frac{2 n-1}{3}$. 

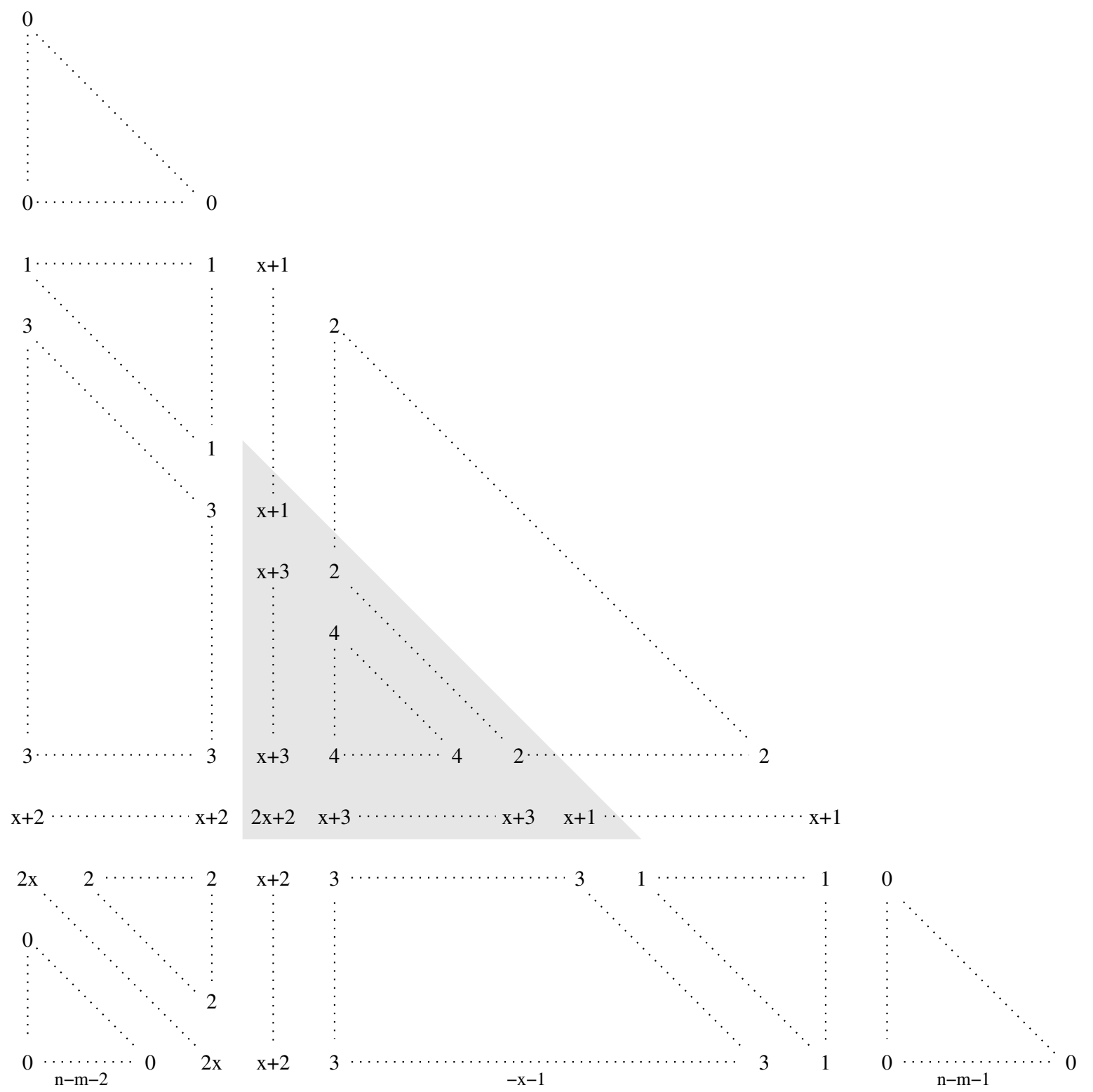

Figure 11: Right type/even, large m: $w(n, m)^{+}$with $x=n-2 m$ and $\frac{2 n-1}{3}<m \leq$ $n-1$.(The case $m=n-1$ reduces to the shaded triangle in the middle.) 

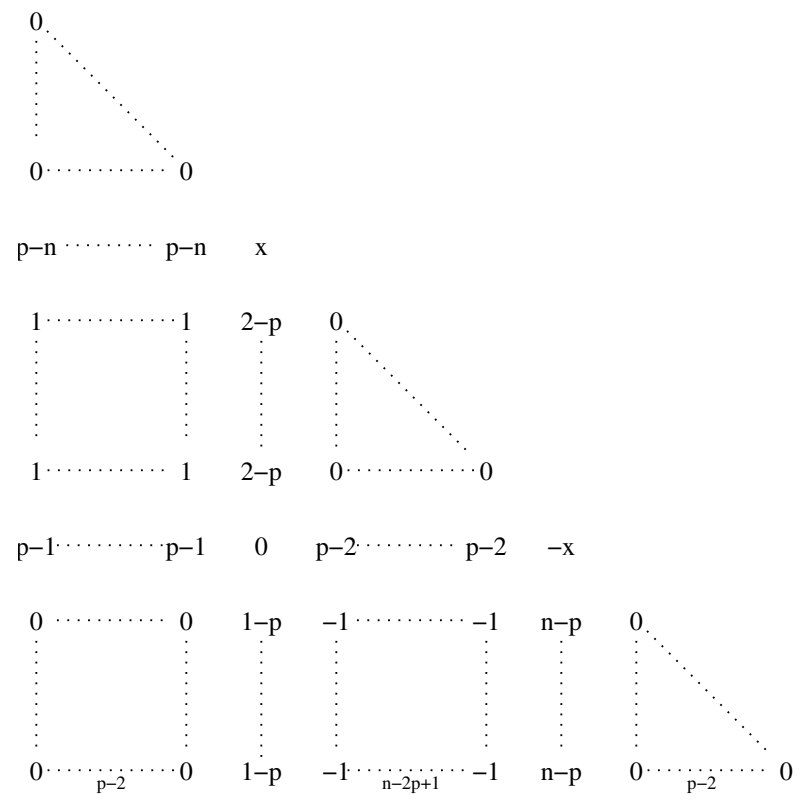

Figure 12: $z(n, p)^{-}$with $x=(p-2)(n-p)$ and $3 \leq p \leq \frac{n+1}{2}$

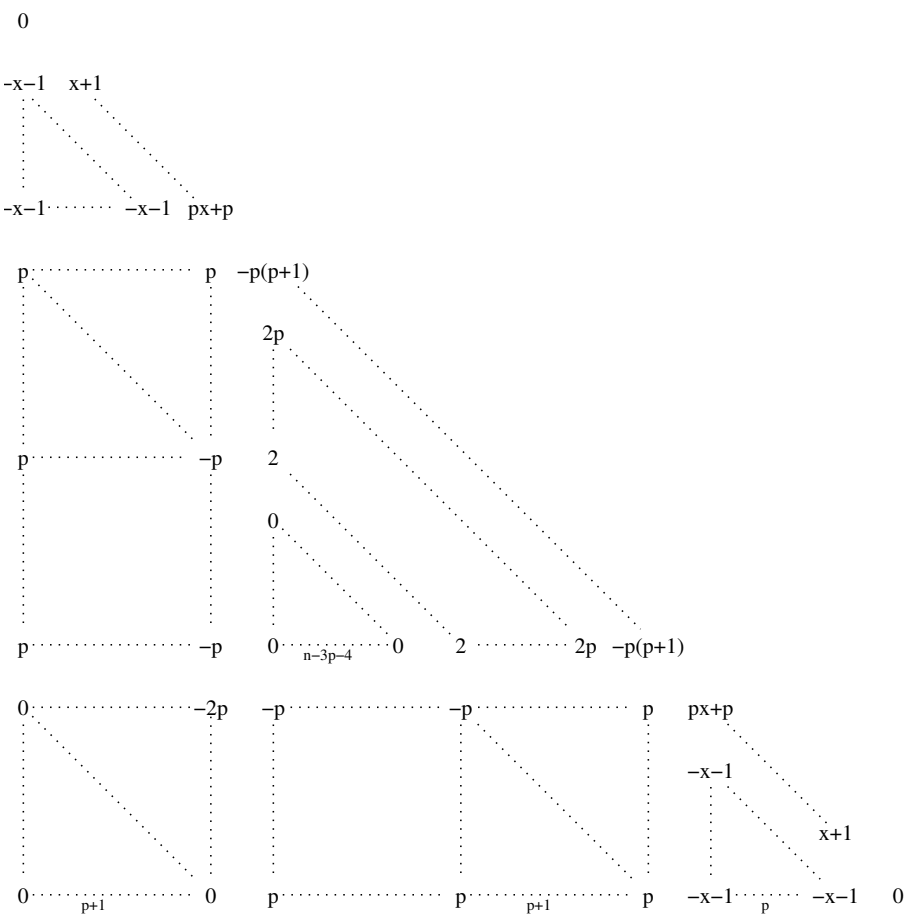

Figure 13: $z(n, p)^{+}$with $x=n-2 p-4$ and $1 \leq p \leq \frac{n-4}{3}$ 


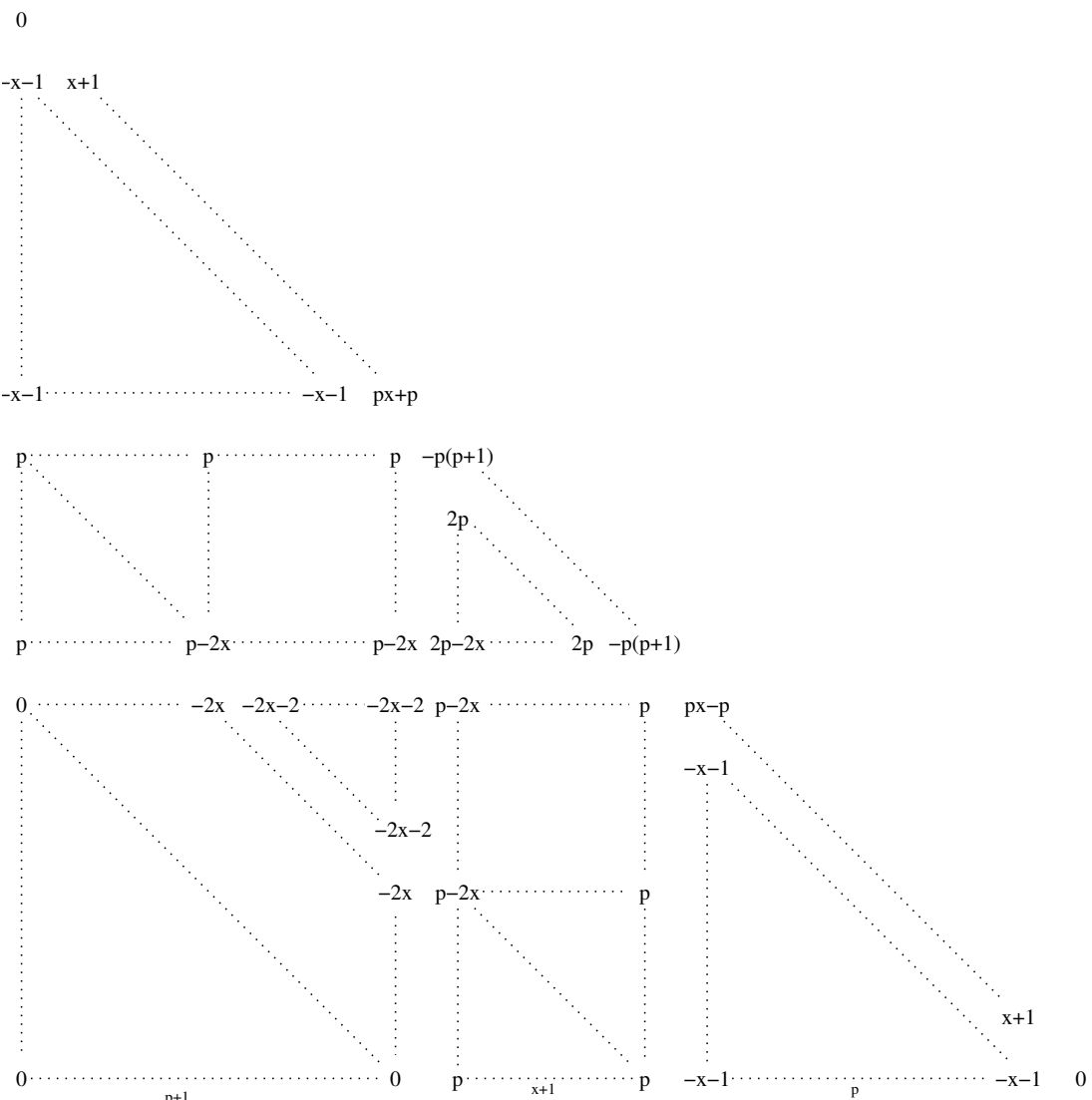

Figure 14: $z(n, p)^{+}$with $x=n-2 p-4$ and $\frac{n-4}{3} \leq p \leq \frac{n-4}{2}$ 


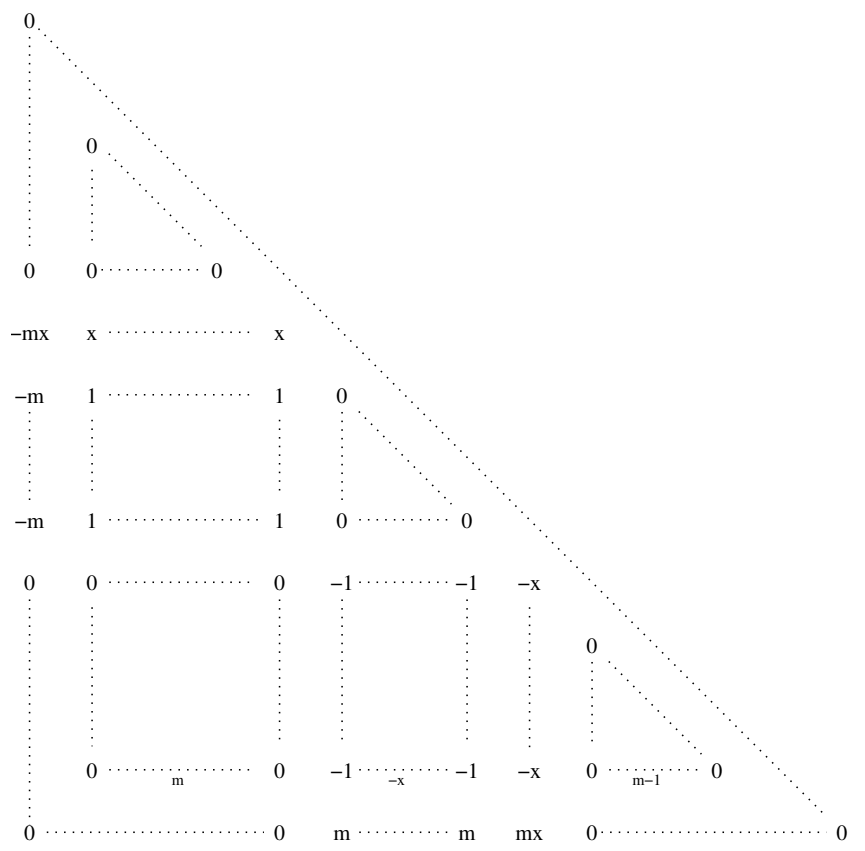

Figure 15: Left type/odd: $\rho^{1} w(n, m)^{-}$with $x=1+2 m-n$ and $0<m<\frac{n}{2}-1$

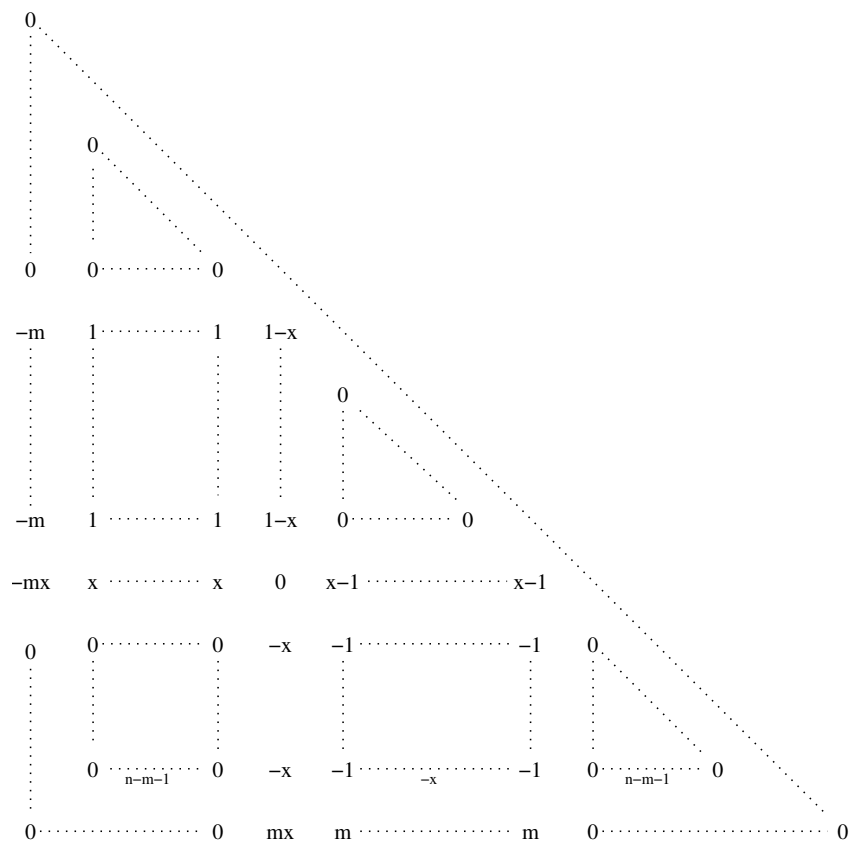

Figure 16: Right type/odd: $\rho^{1} w(n, m)^{-}$with $x=n-2 m$ and $\frac{n+1}{2} \leq m \leq n-1$. 


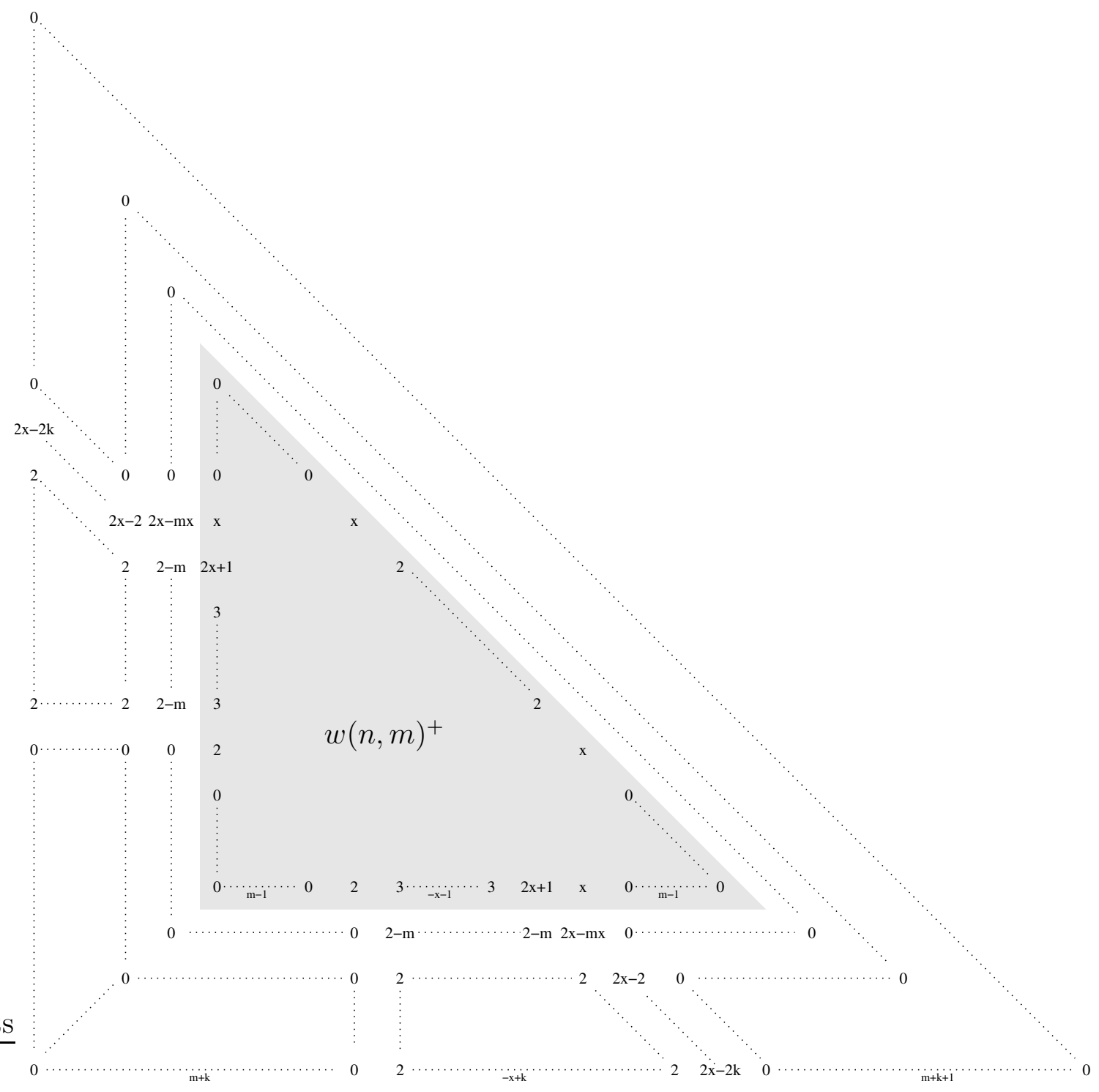

Figure 17: Left type/even: $\rho^{k+1} w(n, m)^{+}$with $x=1+2 m-n$ and $0 \leq m<\frac{n}{2}-1$. 


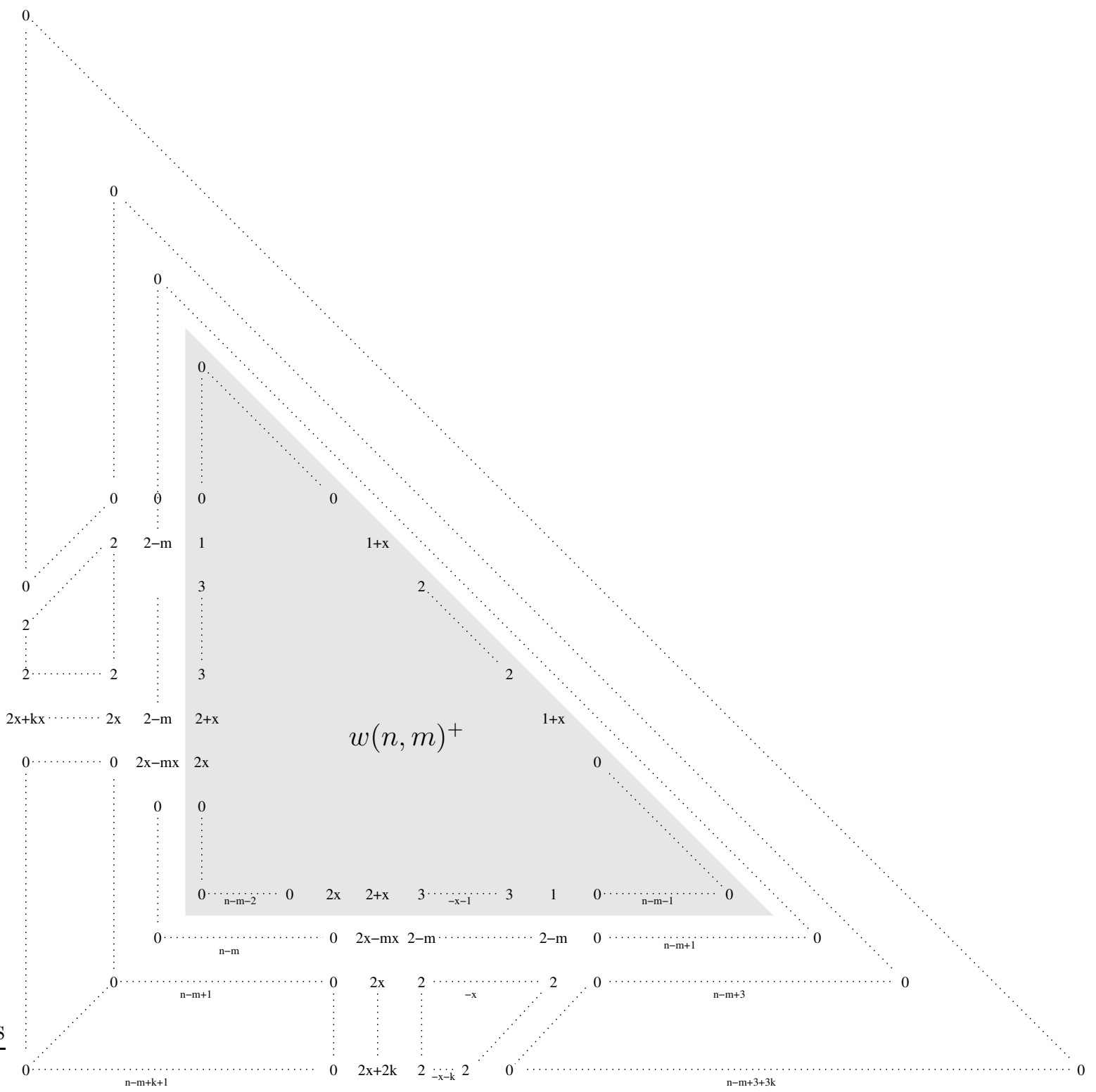

Figure 18: Right type/even: $\rho^{k+2} w(n, m)^{+}$with $x=n-2 m$ and $\frac{n+1}{2} \leq m \leq n-1$. 


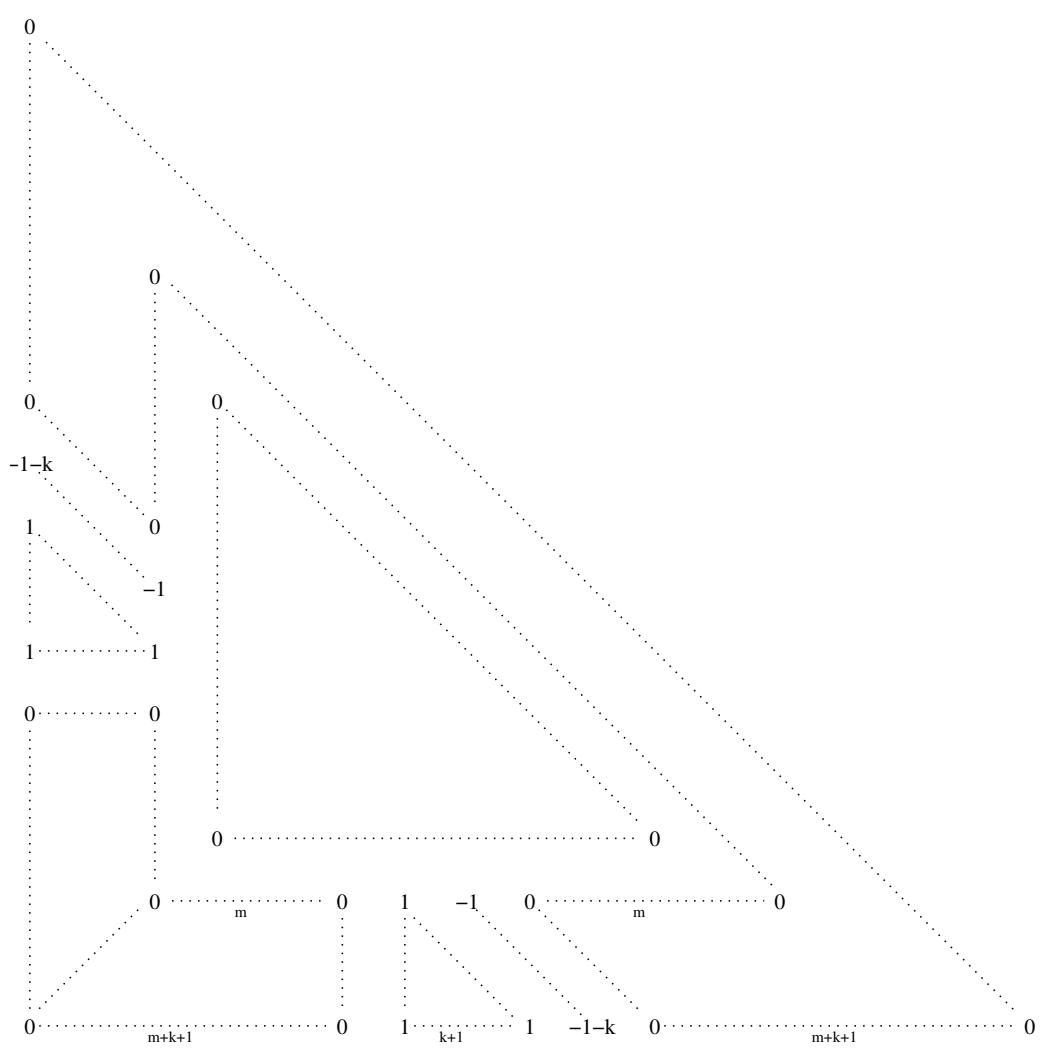

Figure 19: $\rho^{k} y(n)^{+}$with $n$ odd and $m=\frac{n-3}{2}$ 